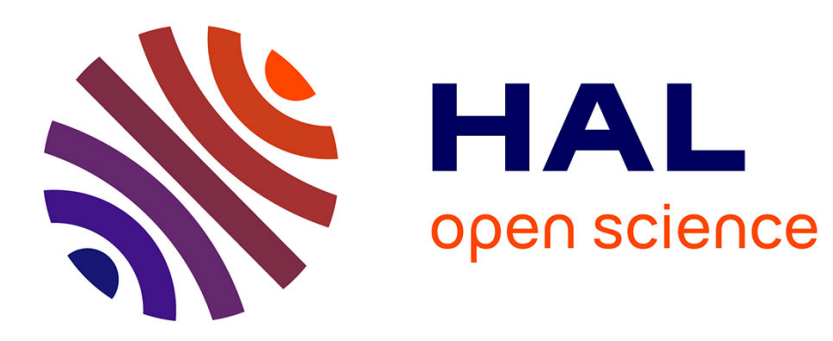

\title{
Discrete Duality Finite Volume Method Applied to Linear Elasticity
}

Benjamin Martin, Frédéric Pascal

\section{To cite this version:}

Benjamin Martin, Frédéric Pascal. Discrete Duality Finite Volume Method Applied to Linear Elasticity. Finite Volumes for Complex Applications VI Problems \& Perspectives, 2011, 4, pp 663-671. 10.1007/978-3-642-20671-9_70 . hal-00798772

\section{HAL Id: hal-00798772 \\ https://hal.science/hal-00798772}

Submitted on 10 Mar 2013

HAL is a multi-disciplinary open access archive for the deposit and dissemination of scientific research documents, whether they are published or not. The documents may come from teaching and research institutions in France or abroad, or from public or private research centers.
L'archive ouverte pluridisciplinaire $\mathbf{H A L}$, est destinée au dépôt et à la diffusion de documents scientifiques de niveau recherche, publiés ou non, émanant des établissements d'enseignement et de recherche français ou étrangers, des laboratoires publics ou privés. 


\title{
Discrete Duality Finite Volume Method Applied to Linear Elasticity
}

Benjamin Martin and Frédéric Pascal

\begin{abstract}
We present the Discrete Duality Finite Volume method (DDFV) for solving the linear elasticity problem on unstructured mesh applied to solids undergoing mechanical loads. The procedure is described in detail for three dimensional problems and some theoretical results are provided: the discrete problem is well-posed, stable and convergent. A number of numerical test problems demonstrates the ability of this finite volume scheme to approach the solution and some comparisons with the conventional finite element method are provided.
\end{abstract}

Key words: finite volume methods, linear elasticity, stability and convergence MSC2010: 65N08, 73C02, 65N12

\section{Motivation}

The finite volume method is extensively used in computational fluid dynamics, on its part the finite element method is the conventional tool for solving solid mechanics. However there is a multitude of physical problems combining fluid and solid mechanics where finite volume methods appear to be a pertinent alternative. Let us quote for instance fluid-structure interaction, deformation of geomechanical reservoir, or even the frost heave problem in freezing soils where the moving frozen fringe introduces a discontinuity in the physical parameters. The finite volume approach for elasticity problems has already been discussed and published in [3], [15], [17], [18] for cell-vertex formulations, in [12] for cell centered formulation with a

Benjamin Martin

CMLA, ENS de Cachan, CNRS, 61 Avenue du Pt Wilson, 94235 Cachan, France e-mail: benjamin.martin@cmla.ens-cachan.fr

Frédéric Pascal

CMLA, ENS de Cachan, CNRS, 61 Avenue du Pt Wilson, 94235 Cachan, France e-mail: frederic.pascal@cmla.ens-cachan.fr 
decoupled strategy for each component, in [6] and [7] for a coupled cell-center version. In this study, we address the DDFV implementation for solving linear elasticity. Let us recall that the principle of the DDFV discretization consists in integrating the system both over a given primal mesh and a dual mesh built from the primal one. Presentation, convergence analysis and numerical tests of DDFV for diffusion, convection-diffusion and Stokes problems are available in [1], [2], [4], [8], [10], [11], [13], [14].

We limit ourselves to the simplest mathematical model of a linear elastic solid which consists in finding the displacement $\mathbf{u} \in \mathbb{R}^{3}$ such that

$$
-\operatorname{div} \sigma(\mathbf{u})=\mathbf{f} \text { on } \Omega, \quad \mathbf{u}=\mathbf{g} \text { on } \Gamma_{D}, \quad \sigma(\mathbf{u}) \cdot \mathbf{n}=\mathbf{h} \text { on } \Gamma_{N}
$$

where $\mathbf{n}$ is the outward normal, $\partial \Omega=\Gamma_{D} \cup \Gamma_{N}$ and where the stress tensor $\sigma$ depends on $\mathbf{u}$ by the Hooke relation that links the strain tensor and the trace of the gradient

$$
\sigma(\mathbf{u})=\lambda \mathbb{D} \text { ivu }+2 \mu \mathbb{D} \mathbf{u} \quad \text { with } \quad \mathbb{D} \mathbf{u}=\frac{\nabla \mathbf{u}+(\nabla \mathbf{u})^{T}}{2} \quad \text { and } \quad \mathbb{D i v u}=\operatorname{div} \mathbf{u} \text { Id } .
$$

For sake of clarity, we assume that $\Omega$ is a bounded polyhedral subset of $\mathbb{R}^{3}$ and that the Lamé coefficients $\lambda$ and $\mu$ are constant.

\section{Finite volume discretization}

A mesh of $\Omega$ is defined by the three sets $\left\{\mathfrak{M}, \mathfrak{M}^{*}, \mathfrak{D}\right\}$, corresponding to the primal, dual and diamond mesh. They form a non overlapping partition of $\Omega$, so that

$$
\bar{\Omega}=\bigcup_{\mathrm{D} \in \mathcal{D}} \mathrm{D}=\bigcup_{\mathrm{K} \in \mathfrak{M}} \mathrm{K}=\frac{1}{2} \bigcup_{\mathrm{K}^{*} \in \mathfrak{M}^{*}} \mathrm{~K}^{*} .
$$

The set $\mathfrak{M}$ is a conforming triangulation of tetraedra. Each element $\mathrm{K}$ in $\mathfrak{M}$ is supplied with a center $\mathbf{x}_{\mathrm{K}}$, in practice the barycenter of $\mathrm{K}$ and $\partial \mathfrak{M}$ denotes the set of faces on the boundary of the domain. The elements of $\mathfrak{M}^{*}$ are polygons $\mathrm{K}^{*}$ corresponding to the primal mesh vertices $\mathbf{x}_{\mathrm{K}^{*}}$. These polygons are the union of all tetrahedra spanned, for each faces $s=\mathrm{K} \cap \mathrm{L}$ or $s=\mathrm{K} \cap \partial \Omega$ having $\mathbf{x}_{\mathrm{K}^{*}}$ as vertex, by $\mathbf{x}_{\mathrm{K}^{*}}$ himself, $\mathbf{x}_{\mathrm{K}}$ or $\mathbf{x}_{\mathrm{L}}$ if it exists, $\mathbf{x}_{s}$ the center of the face $s$, and one of the other vertices of the face $s$. In order to take into account the boundary conditions, the dual mesh is splitted into the internal volumes and the boundary ones corresponding to vertices on the boundary: $\mathfrak{M}^{*}=\mathfrak{M}^{* i} \cup \mathfrak{M}^{* b}$. On its side, diamond cell D in $\mathfrak{D}$ associated to the internal face $s=\mathrm{K} \cap \mathrm{L}$ is the union of the two tetrahedra $\mathrm{D}_{\mathrm{K}, s}$ and $\mathrm{D}_{\mathrm{L}, s}$ spanned by the face $s$ and respectively by the centers $\mathbf{x}_{\mathrm{K}}$ and $\mathbf{x}_{\mathrm{L}}$ (see Figure 1a). For the boundary face $s=\mathrm{K} \cap \partial \Omega$, the corresponding diamond cell is reduced to the tetrahedron $\mathrm{D}_{\mathrm{K}, s}$. The number of primal and dual cells is denoted by $\tau$ and the number of diamond cell by $\delta$. 


\subsection{Discrete operators}

The idea of the DDFV discretization is to construct gradient and divergence operators that are under discrete duality relation by a formula that mimics the Green fomula for continuous functions (see for instance [5] for a detailed construction). A discrete unknown $\mathbf{u}_{\mathrm{K}}$ (resp. $\mathbf{u}_{\mathrm{K}^{*}}$ ) is associated to each volume $\mathrm{K}$ (resp. $\mathrm{K}^{*}$ ) of the primal mesh (resp. dual mesh). They are gathered and denoted by

$$
\mathbf{u}^{\tau}=\left(\mathbf{u}_{\mathrm{K}}, \mathbf{u}_{\mathrm{K}^{*}}\right)_{\mathrm{K} \in \mathfrak{M}, \mathrm{K}^{*} \in \mathfrak{M}^{*}} .
$$

For a vector field $\mathbf{u}^{\tau}$ in $\left(\mathbb{R}^{d}\right)^{\tau}$, we define on each diamond cell a consistent discrete gradient operator $\nabla^{\mathfrak{D}} \mathbf{u}^{\tau}=\left(\nabla^{\mathrm{D}} \mathbf{u}^{\tau}\right)_{\mathrm{D} \in \mathfrak{D}}$ in $\left(\mathscr{M}_{d}(\mathbb{R})\right)^{\delta}$ and a consistent discrete divergence operator $\operatorname{div}^{\mathfrak{D}} \mathbf{u}^{\tau}=\left(\operatorname{div}^{\mathrm{D}} \mathbf{u}^{\tau}\right)_{\mathrm{D} \in \mathfrak{D}}$ in $\mathbb{R}^{\delta}$ such that on the internal face $s=\mathrm{K} \cap \mathrm{L}$ and for the associated diamond cell $\mathrm{D}=\mathrm{D}_{\mathrm{K}, s} \cup \mathrm{D}_{\mathrm{L}, s}$, the gradient is given by $\nabla^{\mathrm{D}} \mathbf{u}^{\tau}=\frac{\left|\mathrm{D}_{\mathrm{K}, s}\right|}{\mathrm{D} \mid} \nabla^{\mathrm{D}_{\mathrm{K}, s}} \mathbf{u}^{\tau}+\frac{\left|\mathrm{D}_{\mathrm{L}, s}\right|}{\mathrm{D} \mid} \nabla^{\mathrm{D}_{\mathrm{L}, s}} \mathbf{u}^{\tau}$ and the divergence by

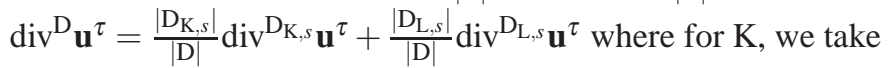

$$
\begin{aligned}
\nabla^{\mathrm{D}_{\mathrm{K}, s}} \mathbf{u}^{\tau} & =\frac{1}{3\left|\mathrm{D}_{\mathrm{K}, s}\right|}\left(\mathbf{u}_{s}-\mathbf{u}_{\mathrm{K}}\right) \otimes \mathbf{N}_{\mathrm{K} s}+\frac{1}{3\left|\mathrm{D}_{\mathrm{K}, s}\right|} \sum_{i=1}^{d} \mathbf{u}_{i} \otimes\left(\mathbf{N}_{i-1}-\mathbf{N}_{i+1}\right) \\
\operatorname{div}^{\mathrm{D}_{\mathrm{K}, s} \mathbf{u}^{\tau}} & =\frac{1}{3\left|\mathrm{D}_{\mathrm{K}, s}\right|}\left(\mathbf{u}_{s}-\mathbf{u}_{\mathrm{K}}\right) \cdot \mathbf{N}_{\mathrm{K} s}+\frac{1}{3\left|\mathrm{D}_{\mathrm{K}, s}\right|} \sum_{i=1}^{d} \mathbf{u}_{i} \cdot\left(\mathbf{N}_{i-1}-\mathbf{N}_{i+1}\right)
\end{aligned}
$$

Here $|\cdot|$ denotes the measure and $\left(\mathbf{x}_{i}\right)_{i=1}^{d}$, respectively $\left(\mathbf{u}_{i}\right)_{i=1}^{d}$, the vertices of the

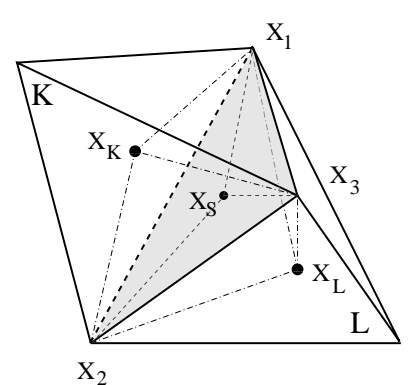

(a)

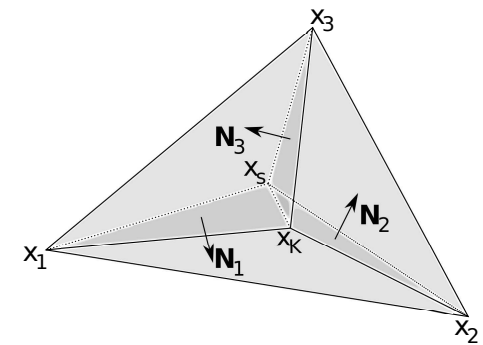

(b)

Fig. 1 (a) Primal and diamond cell - (b) Normal orientations in the diamond cell

face $s$, respectively the corresponding unknowns, with the local numbering convention $\mathbf{x}_{0}=\mathbf{x}_{d}$. The outward normals are defined by (see Figure 1b) 


$$
\begin{aligned}
& \mathbf{N}_{\mathrm{K} s}=\sum_{i=1}^{d} \mathbf{N}_{s, i-1, i} \quad \text { with } \quad \mathbf{N}_{s, i-1, i}=\frac{1}{2}\left(\mathbf{x}_{i}-\mathbf{x}_{s}\right) \wedge\left(\mathbf{x}_{i-1}-\mathbf{x}_{s}\right) \\
& \mathbf{N}_{i}=\frac{1}{2}\left(\mathbf{x}_{\mathrm{K}}-\mathbf{x}_{s}\right) \wedge\left(\mathbf{x}_{i}-\mathbf{x}_{s}\right)
\end{aligned}
$$

and $\mathbf{u}_{s}$ is chosen in order to satisfy the continuity of fluxes (see 7). Otherwise, on a boundary face $s \in \partial \mathfrak{M}$ and for the corresponding diamond cell $\mathrm{D}=\mathrm{D}_{\mathrm{K}, s}$, the gradient and the divergence are simply $\nabla^{\mathrm{D}} \mathbf{u}^{\tau}=\nabla^{\mathrm{D}_{\mathrm{K}, s}} \mathbf{u}^{\tau}$ and $\operatorname{div}^{\mathrm{D}} \mathbf{u}^{\tau}=\operatorname{div}^{\mathrm{D}_{\mathrm{K}, s} \mathbf{u}^{\tau}}$ but $\mathbf{u}_{s}$ depending on the boundary datas is explicited in (8).

\subsection{The DDFV scheme}

For $\mathbf{u}^{\tau}$ in $\left(\mathbb{R}^{d}\right)^{\tau}$, we are now able to define the discrete strain tensor $\mathbb{D}^{\mathfrak{D}} \mathbf{u}^{\tau}=$ $\left(\mathbb{D}^{\mathrm{D}} \mathbf{u}^{\tau}\right)_{\mathrm{D} \in \mathfrak{D}}$ and the divergence one $\mathbb{D i v}^{\mathfrak{D}} \mathbf{u}^{\tau}=\left(\mathbb{D i v}^{\mathrm{D}} \mathbf{u}^{\tau}\right)_{\mathrm{D} \in \mathfrak{D}}$ by

$$
\mathbb{D}^{\mathrm{D}} \mathbf{u}^{\tau}=\frac{\nabla^{\mathrm{D}} \mathbf{u}^{\tau}+\left(\nabla^{\mathrm{D}} \mathbf{u}^{\tau}\right)^{T}}{2} \quad, \quad \mathbb{D i v}^{\mathrm{D}} \mathbf{u}^{\tau}=\operatorname{div}^{\mathrm{D}} \mathbf{u}^{\tau} \mathrm{Id} \quad \forall \mathrm{D} \in \mathfrak{D} .
$$

After extending this definition to each tetrahedron that composes the diamond cell, we can specify that the displacement $\mathbf{u}_{s}$ at an internal face $s=\mathrm{K} \cap \mathrm{L}$ has to satisfy the continuity of the fluxes

$$
\left(\lambda \mathbb{D i v}^{\mathrm{D}_{\mathrm{K}, s}} \mathbf{u}^{\tau}+2 \mu \mathbb{D}^{\mathrm{D}_{\mathrm{K}, s} \mathbf{u}^{\tau}}\right) \mathbf{N}_{\mathrm{K} s}=-\left(\lambda \mathbb{D i v}^{\mathrm{D}_{\mathrm{L}, s}} \mathbf{u}^{\tau}+2 \mu \mathbb{D}^{\mathrm{D}_{\mathrm{L}, s}} \mathbf{u}^{\tau}\right) \mathbf{N}_{\mathrm{L} s} .
$$

Now for a tensor field $\xi^{\mathfrak{D}}$ in $\left(\mathscr{M}_{d}(\mathbb{R})\right)^{\delta}$, we define a consistent approximation of the discrete divergence operator equal to

$$
\left(\operatorname{div}^{\mathfrak{M}} \xi^{\mathfrak{D}}, \operatorname{div}^{\mathfrak{M}} \xi^{\mathfrak{D}}\right)=\left(\left(\operatorname{div}^{\mathrm{K}} \xi^{\mathfrak{D}}\right)_{\mathrm{K} \in \mathfrak{M}},\left(\operatorname{div}^{\mathrm{K}^{*}} \xi^{\mathfrak{D}}\right)_{\mathrm{K}^{*} \in \mathfrak{M}^{*}}\right)
$$

with

$$
\operatorname{div}^{\mathrm{K}} \xi^{\mathfrak{D}}=\frac{1}{|\mathrm{~K}|} \sum_{s \in \partial \mathrm{K}} \xi^{\mathrm{D}} \mathbf{N}_{\mathrm{K} s} \quad \text { and } \quad \operatorname{div}^{\mathrm{K}^{*}} \xi^{\mathfrak{D}}=\frac{1}{\left|\mathrm{~K}^{*}\right|} \sum_{s \ni \mathbf{x}_{\mathrm{K}^{*}}} \xi^{\mathrm{D}} \mathbf{N}_{\mathrm{K}^{*} s}
$$

and where $\mathrm{D}$ is the diamond cell associated to the face $s . \mathbf{N}_{\mathrm{K}^{*} s}$ is the normal to $\partial \mathrm{K}^{*}$ pointing outward $\mathrm{K}^{*}$ and it can be explicited using local numbering and applying formula (5):

$$
\mathbf{N}_{\mathrm{K}^{*} s}=\left\{\begin{array}{l}
\mathbf{N}_{i+1}^{K}-\mathbf{N}_{i-1}^{K}+\mathbf{N}_{i+1}^{L}-\mathbf{N}_{i-1}^{L} \text { for an internal face } s=\mathrm{K} \cap \mathrm{L} \\
\mathbf{N}_{i+1}-\mathbf{N}_{i-1}+\mathbf{N}_{s, i-1, i}+\mathbf{N}_{s, i, i+1} \text { for a boundary face } s=\mathrm{K} \cap \partial \Omega
\end{array}\right.
$$

where we assume that $x_{\mathrm{K}^{*}}=x_{i}^{\mathrm{K}}\left(\operatorname{resp} . x_{\mathrm{K}^{*}}=x_{i}^{\mathrm{L}}\right)$ in the volume $\mathrm{K}(\operatorname{resp} . \mathrm{L})$.

Let us now denote $\mathbf{f}^{\mathfrak{M}}=\left(\mathbf{f}^{\mathrm{K}}\right)_{\mathrm{K} \in \mathfrak{M}}$ and $\mathbf{f}^{\mathfrak{M}^{* i}}=\left(\mathbf{f}^{\mathrm{K}^{*}}\right)_{\mathrm{K}^{*} \in \mathfrak{M}^{* i}}$, where $\mathbf{f}^{\mathrm{K}}$ and $\mathbf{f}^{\mathrm{K}^{*}}$ are the average of the external force $\mathbf{f}$ on primal and dual cells. Then the DDFV scheme, written here, for sake of simplicity, only for displacement boundary condi- 
tions, consists in finding $\mathbf{u}^{\tau} \in\left(\mathbb{R}^{d}\right)^{\tau}$ such that

$$
\left\{\begin{array}{l}
-\operatorname{div}^{\mathfrak{M}}\left(\lambda \mathbb{D i v}^{\mathfrak{D}} \mathbf{u}^{\tau}+2 \mu \mathbb{D}^{\mathfrak{D}} \mathbf{u}^{\tau}\right)=\mathbf{f}^{\mathfrak{M}} \\
-\operatorname{div}^{\mathfrak{M}^{* i}}\left(\lambda \mathbb{D i v}^{\mathfrak{D}} \mathbf{u}^{\tau}+2 \mu \mathbb{D}^{\mathfrak{D}} \mathbf{u}^{\tau}\right)=\mathbf{f}^{\mathfrak{M} * i} \\
\mathbf{u}_{s}=\mathbf{g}\left(\mathbf{x}_{s}\right), \quad \forall s \in \partial \mathfrak{M} \\
\mathbf{u}_{\mathrm{K}^{*}}=\mathbf{g}\left(\mathbf{x}_{\mathrm{K}^{*}}\right), \quad \forall \mathrm{K}^{*} \in \mathfrak{M}^{* b}
\end{array}\right.
$$

\subsection{Existence, stability and convergence results}

Applying discrete Green formula, Korn and Poincaré inequalities, divergence equality and approximation results on the center value projection operator (see [14]), we prove that the numerical scheme is well-posed, stable and convergent:

Theorem 1. Under the assumption that mes $\left(\Gamma_{D}\right) \neq 0$, the DDFV scheme for linear elasticity (8) yields to a symmetric positive definite system of linear equations. So it admits exactly one solution $\mathbf{u}^{\tau} \in\left(\mathbb{R}^{d}\right)^{\tau}$

Theorem 2. Let $\mathbf{u}^{\tau} \in\left(\mathbb{R}^{d}\right)^{\tau}$ be the solution of the discrete problem (8). Then there exists a constant $C$ depending only on the regularity of the mesh such that

$$
\mu\left\|\nabla^{\mathfrak{D}} \mathbf{u}^{\tau}\right\|_{2}^{2}+\frac{\lambda}{3}\left\|\mathbb{D} i v^{\mathfrak{D}} \mathbf{u}^{\tau}\right\|_{2}^{2} \leq C\left\|\mathbf{f}^{\tau}\right\|_{2}^{2}
$$

Theorem 3. Assuming that the exact solution of the continuous problem (1) is regular enough then there exists a constant $C$ depending only on the regularity of the mesh, such that

$$
\left\|\mathbf{u}-\mathbf{u}^{\tau}\right\|_{2}+\left\|\nabla \mathbf{u}-\nabla^{\mathfrak{D}} \mathbf{u}^{\tau}\right\|_{2} \leq C \operatorname{size}(\mathfrak{M})
$$

\section{Numerical experiments}

The DDFV method has been implemented in two and three dimensions. Free and imposed traction conditions (described in [16]) are also taken into account. Both homogeneous and non homogeneous test cases are considered. Comparisons are made with the analytical solution or with the clasical finite element one.

\subsection{Two dimensional examples}

Following a study of [9], we apply the code to a simple test case with analytical solution in order to study the convergence properties. The geometry of the homogeneous square plate and the specified boundary conditions are shown in Figure 2(a). Lamé 


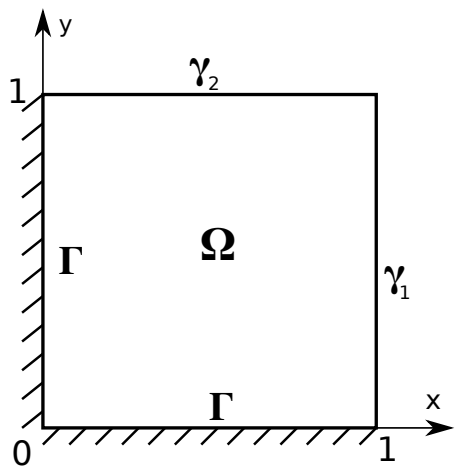

(a)

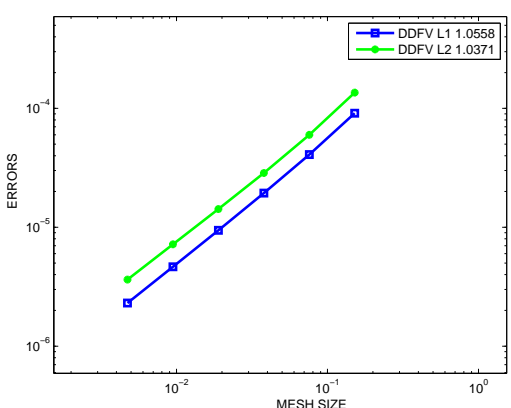

(b)

Fig. 2 (a) Geometry and test setup - (b) $L^{1}$ and $L^{2}$ nors of the error between the analytical and the numerical displacement.

coefficients $(\lambda, \mu)=\left(2.910^{9}, 1.910^{9}\right)$ correspond to Young modulus and Poisson ratio $(E, v)=\left(510^{9}, 0.3\right)$. The displacement $\mathbf{g}$ is null on $\Gamma$ boundary and the traction is imposed on $\gamma_{1}$ and $\gamma_{2}$ boundaries:

$$
\mathbf{g}_{\mid \gamma_{1}}=\left(\begin{array}{l}
((2 \mu+\lambda) y-2 \lambda) 10^{-2} \\
\mu(1-2 y) 10^{-2}
\end{array}\right) \quad \mathbf{g}_{\mid \gamma_{2}}=\left(\begin{array}{l}
\mu(x-2) 10^{-2} \\
(-2(2 \mu+\lambda) x+\lambda) 10^{-2}
\end{array}\right) .
$$

The external force is equal to $\mathbf{f}=(\mu+\lambda) 10^{-2}(2,-1)$ and the corresponding exact displacement is $\mathbf{u}=x y 10^{-2}(1,-2)$. The comparison between the analytical and numerical displacement obtained for various primal meshes are plotted in Figure 2(b) with an order of convergence of one.

The second example concerns a domain with non homogeneous material properties. The plate (without deformation) is composed of the part $[0,3] \times[0,1]$ with a hole inside and $(\lambda, \mu)=(5.6,2.6)$ and the part $[3,4] \times[0,1]$ with $(\lambda, \mu)=(10,8)$. Null displacement is imposed on the left side of the domain, a load of 1 (resp. a displacement of 1) is imposed on the right side for Figure 3 (resp. for Figure 4). There is a free traction elsewhere. The deformed domain obtained with the present scheme (above) and with the conventional finite element method (below) are plotted. In both case, solution are similar, the largest differences are observed in the load one.

\subsection{Three dimensional test}

The domain is the unit cube with an embedding condition on the bottom $(z=0)$, imposed displacement $(0,0,-0.5)$ on the top $(z=1)$ simulating a compression of the domain (see Figure $5 \mathrm{~b}$ ) and free traction conditions on the vertical sides of the cube. For Lamé coefficients $(\lambda, \mu)=(28.8,19.2)$, the solution is compared with the P1 finite element one on a series of meshes: Figure 5a shows the behavior of the 

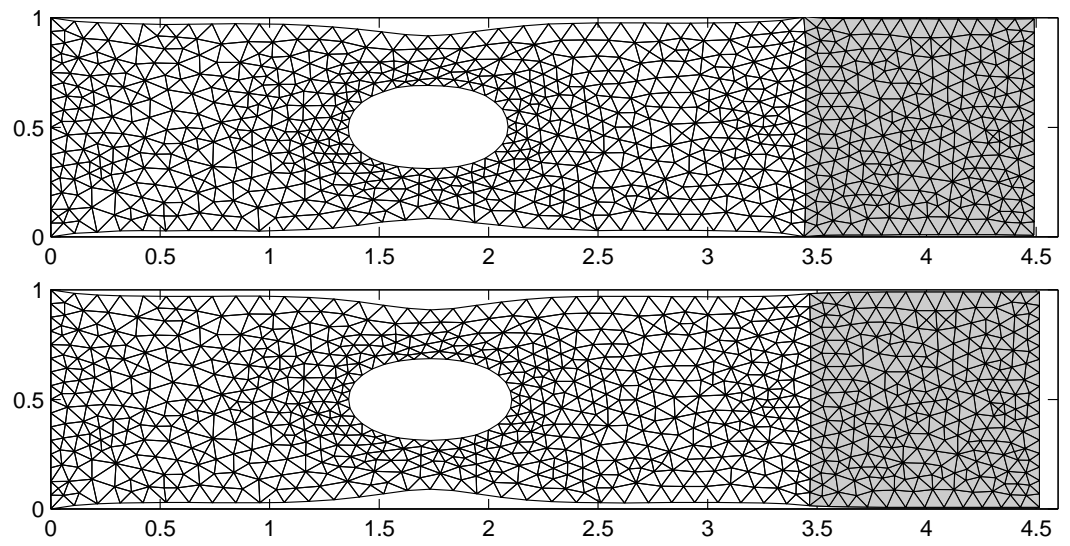

Fig. 3 Deformed domain for the non homogeneous case with an imposed load on the right. DDFV above and FE below
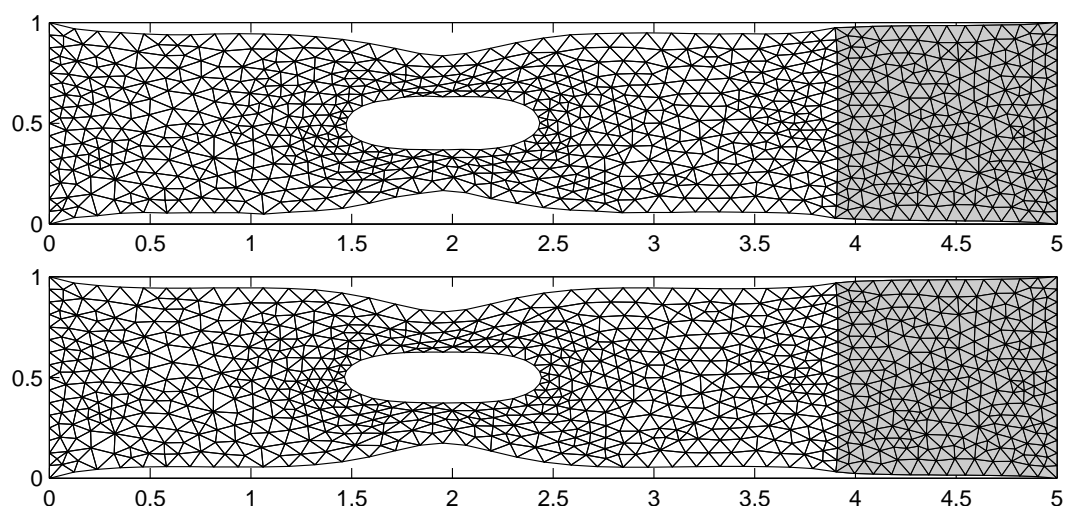

Fig. 4 Deformed domain for the non homogeneous case with an imposed displacement on the right. DDFV above and FE below

error in $L^{2}$ and $L^{1}$ norms and reveals that the DDFV solution of the linear elasticity problem converges as we expect.

\section{References}

1. Andreianov, B., Bendahmane, M., Karlsen, K., Pierre, C.: Convergence of discrete duality finite volume schemes for the cardiac bidomain model. Arxiv preprint arXiv:1010.2718 (2010)

2. Andreianov, B., Boyer, F., Hubert, F.: Discrete duality finite volume schemes for Leray-Lionstype elliptic problems on general 2D meshes. Numer. Methods Partial Differential Equations 23(1), 145-195 (2007)

3. Bailey, C., Cross, M.: A finite volume procedure to solve elastic solid mechanics problems in three dimensions on an unstructured mesh. Int. J. Numer. Methods Eng. 38(10), 1757-1776 


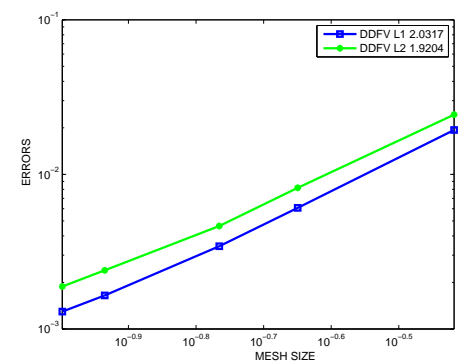

(a)

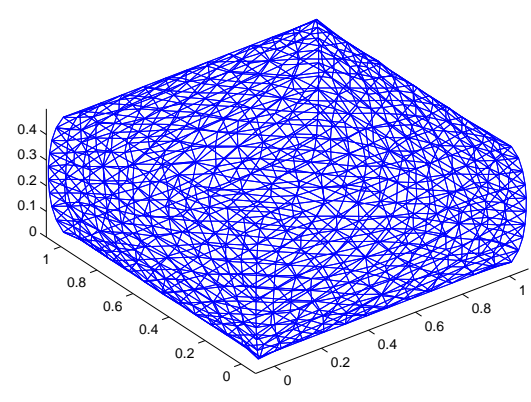

(b)

Fig. 5 (a) Differences with the finite element solution - (b) DDFV deformed domain

(1995)

4. Coudière, Y., Manzini, G.: The discrete duality finite volume method for convection-diffusion problems. SIAM J. Numer. Anal. 47(6), 4163-4192 (2010)

5. Coudière, Y., Pierre, C., Rousseau, O., Turpault, R.: A 2D/3D discrete duality finite volume scheme. Application to ECG simulation. Int. J. Finite Vol. 6(1), 24 (2009)

6. Demirdžić, I., Muzaferija, S.: Finite volume method for stress analysis in complex domains. Int. J. Numer. Methods Eng. 37(21), 3751-3766 (1994)

7. Demirdžić, I., Muzaferija, S., Perić, M.: Benchmark solutions of some structural analysis problems using finite-volume method and multigrid acceleration. Int. J. Numer. Methods Eng. 40(10), 1893-1908 (1997)

8. Domelevo, K., Omnes, P.: A finite volume method for the laplace equation on almost arbitrary two-dimensional grids. M2AN Math. Model. Numer. Anal. 39, 1203-1249 (2005)

9. Figueiredo, J., Viano, J.: Finite elements q1-lagrange for the linear elasticity problem. Tech. rep., Universidade de Santiago de Compostela (2005)

10. Herbin, R., Hubert, F.: Benchmark on discretization schemes for anisotropic diffusion problems on general grids. In: Finite volumes for complex applications V, pp. 659-692. ISTE, London (2008)

11. Hermeline, F.: A finite volume method for the approximation of diffusion operators on distorted meshes. J. Comput. Phys. 160(2), 481-499 (2000)

12. Jasak, H., Weller, H.: Application of the finite volume method and unstructured meshes to linear elasticity. Int. J. Numer. Methods Eng. 48(2), 267-287 (2000)

13. Krell, S.: Stabilized DDFV schemes for stokes problem with variable viscosity on general $2 \mathrm{~d}$ schemes. Numer. Methods Partial Differential Equations (2010)

14. Krell, S., Manzini, G.: The discrete duality finite volume method for the stokes equations on 3-d polyhedral meshes (2010). URL http://hal.archives-ouvertes.fr/hal-00448465/en/

15. Maitre, J.F., Rezgui, A., Souhail, H., Zine, A.M.: High order finite volume schemes. Application to non-linear elasticity problems. In: Finite volumes for complex applications, III (Porquerolles, 2002), pp. 391-398. Hermes Sci. Publ., Paris (2002)

16. Martin, B.: Résolution du problème de l'élasticité linéaire en volumes finis. Ph.D. thesis, ENS de Cachan (2011)

17. Souhail, H.: Schéma volumes finis : Estimation d'erreur a posteriori hiérarchique par éléments finis mixtes. Résolution de problèmes d'élasticité non-linéaire. Ph.D. thesis, Ecole Centrale de Lyon (2004). URL http://tel.archives-ouvertes.fr/tel-00005418/en/

18. Wenke, P., Wheel, M.: A finite volume method for solid mechanics incorporating rotational degrees of freedom. Computers \& Structures 81(5), 321-329 (2003)

The paper is in final form and no similar paper has been or is being submitted elsewhere. 